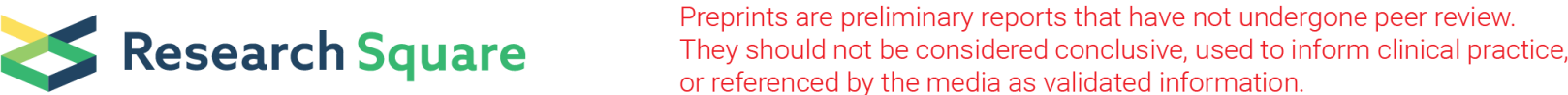

\section{Remifentanil versus combined spinal-epidural technique for labour analgesia in multiparous women: a prospective non-randomized trial}

Tatjana Stopar Pintaric ( $\boldsymbol{D}$ tatjanas38@gmail.com )

Univerza v Ljubljani Medicinska Fakulteta https://orcid.org/0000-0003-2750-5386

Tea Zagar

Univerza v Ljubljani Medicinska Fakulteta

Neli Semrl

Univerza v Ljubljani Medicinska Fakulteta

Nejc Umek

Univerza v Ljubljani Medicinska Fakulteta

Miha Lucovnik

Univerza v Ljubljani Medicinska Fakulteta

Marc Van de Velde

Katholieke Universiteit Leuven

Iva Blajic

Univerzitetni klinicny Center Ljubljana

Research article

Keywords: Labour analgesia; multiparity; combined spinal-epidural analgesia; remifentanil; patientcontrolled analgesia; pregnancy.

Posted Date: March 3rd, 2020

DOI: https://doi.org/10.21203/rs.2.21366/v2

License: (a) (i) This work is licensed under a Creative Commons Attribution 4.0 International License. Read Full License 


\section{Abstract}

Background : Remifentanil has a suitable pharmacological profile for labour analgesia. Fast set up, onset, and limited time efficacy may render it useful in multiparas with a faster labour progression. The objective of this prospective, non-randomized trial was to compare the pain relief for vaginal delivery between remifentanil patient-controlled analgesia (RPCA) with that of combined spinal-epidural analgesia (CSEA) in multiparas. Methods : After making an informed choice of either RPCA or CSEA, 162 multiparous women in the active phase of first-stage labour were enrolled in this study. The primary outcome was pain intensity measured during the whole period of labour using an 11-point numerical rating scale. The secondary outcomes were satisfaction with pain-relief, adverse effects, labour progress and outcomes. Results : CSEA provided lower pain scores and a higher satisfaction rate than RPCA [3.9 (0.36) vs 2.6 (1.1), $P<0.001$ ]. Also, more patients opted for the CSEA for their next labour [CSEA; 77 (95\%) vs. RPCA; 65 (81\%), P =0.003]. However, more than $80 \%$ of women in the RPCA group graded their satisfaction as good and very good with a high rate of recommendation and no crossovers observed. Remifentanil was associated with desaturation, bradypnea and apnoea in 27 (34\%), 17 (21\%) and 20 $(25 \%)$ respective cases. All recovered spontaneously upon verbal or tactile stimulation. No clinically important differences were noted in sedation level, labour progress and outcomes. Conclusions : CSEA provided superior pain relief compared to RPCA in multiparas. Despite that, remifentanil was highly rated, with a high rate of recommendation and no crossovers to CSEA. That can be attributed to the fact that women with previewed shorter labour were more likely to choose a less invasive analgesic option which provided an element of personal control. Thus, given no difference in labour outcomes, remifentanil could be offered as a routine analgesic option provided rigorous adherence to the necessary protocols are ensured. The presence of a midwife is essential for timely management of respiratory depression or other severe outcomes.

\section{Background}

Epidural analgesia is considered the most efficient method of pain relief during labour and is often the preferred analgesic choice. Remifentanil has a suitable pharmacological profile for labour analgesia. Fast set up, rapid availability and short use of pain relief may render it beneficial for women with a faster labour progression $[1,2]$.

Several studies comparing remifentanil patient-controlled analgesia (RPCA) with epidural analgesia observed worse pain scores and a lower satisfaction rate in the remifentanil group [3-6]. In multiparous women, by contrast, a higher satisfaction rate with remifentanil compared to epidural analgesia was reported due to fast onset, rapid availability and short use of pain relief in this group of patients [5]. Since pain intensity was assessed as a secondary outcome [5] and a combined spinal-epidural analgesia (CSEA) proved superior in comparison to epidural technique in multiparous women [7], further studies are needed to compare the analgesic efficacy of remifentanil and CSEA in this group of patients. 
Thus, the primary aim of this study was to compare the pain intensity between remifentanil and CSEA in multiparous women measured during the whole labour using an 11-point numerical rating scale. The secondary outcomes were overall satisfaction with pain-relief, the incidence of adverse effects, labour progress and labour outcomes.

\section{Materials And Methods}

This prospective observational study was approved by the Republic of Slovenia National Medical Ethics Committee (Permit No. 91/04/16) and registered at the central database of ClinicalTrials.gov under the trial ID NCT02963337, (https://clinicaltrials.gov/ct2/show/NCT02963337?term=02963337\&rank=1) on November 15, 2016. The study was conducted from January 2017 to September 2018 in the labour and delivery unit of the Perinatology Department, Division of Obstetrics and Gynaecology with approximately 6000 deliveries per year, neuraxial and remifentanil analgesia rate of $60 \%$ (ratio 1:1) and caesarean section rate of $21 \%$.

Consecutively admitted at the labour and delivery suit, multiparous women with singleton pregnancies in their active phase of first stage labour requesting pain relief were asked to participate in the study. They were informed about the two different analgesic options being studied, i.e. RPCA and CSEA, their advantages and disadvantages. After signing an informed consent, they were allocated either to a RPCA or CSEA group on the basis of their informed choices of labour analgesia. The inclusion criteria were age 18 to 55, American Society of Anaesthesiologists (ASA) physical status 2 or 3, uncomplicated pregnancy at 37 0/7 - $406 / 7$ weeks of gestation, absence of known foetal congenital abnormalities, vertex presentation, ongoing uterine contraction, cervical dilation 3 to $6 \mathrm{~cm}$ and normal cardiotocography (CTG). Exclusion criteria were preeclampsia, contraindications to CSEA (coagulation and neurological abnormalities, infection/fever) and remifentanil (opioid drug abuse, pethidine given within the two previous hours, known allergic reaction to remifentanil, morbid obesity with body mass index (BMI) $>40$ and obstructive sleep apnoea).

In the RPCA group, the women were introduced to the PCA pump (Rythmic ${ }^{\text {TM }}$ Evolution, Micrel Medical Devices, Athens, Greece) and told to use PCA at the start of each uterine contraction [8]. Remifentanil hydrochloride (Ultiva, GlaxoSmithKline, Oslo, Norway) was diluted in saline to a concentration of $40 \mu \mathrm{g}$ $\mathrm{ml}^{-1}$ and administered stepwise from 20 to a maximum of $40 \mu \mathrm{g}$ with a bolus duration of 20 seconds and 2 minutes lockout interval with no background infusion. Dose adjustment was performed by anaesthesiological staff at patients' request. The bolus dose was increased if pain intensity as assessed by an 11-point numerical rating scale (NRS; 0 is no pain and 10 the worst imaginable pain) increased and a patient's respiratory rate was $>9$ breaths $\mathrm{min}^{-1}$, oxygen saturation $\left(\mathrm{SpO}_{2}\right) \geq 94 \%$, heart rate $>50 \mathrm{~min}^{-1}$ and sedation score $\leq 2$ on a five-point categorical scale (scale 1-5: 1=alert, 2=slightly drowsy, 3=drowsy, 4=very drowsy, $5=$ =unarousable) [9-11]. The use of PCA was allowed until 5-10 minutes prior to cord clamping. Women in the RPCA group had one-to-one midwifery care. In accordance with the institutional standard operating protocol, women were continuously monitored with Capnostream $\AA$ capnograph (Oridion ${ }^{\circledR}$, Jeruzalem, Israel) with an oral-nasal cannula, sampling from both the nose and mouth 
(Oridion $\AA)$. Supplemental oxygen $\left(2 I \mathrm{~min}^{-1}\right)$ was given to all patients via a nasal catheter. The respiratory monitor recorded continuous waveform of end tidal $\mathrm{CO}_{2}$, respiratory rate, $\mathrm{SpO}_{2}$ and heart rate with the alarms being activated by oxygen desaturation $\left(\mathrm{SpO}_{2}<94 \%\right)$, bradypnea (respiratory rate $\left.<8 \mathrm{~min}^{-1}\right)$ and apnoea longer than 20 seconds, triggering staged interventions started with a verbal command to take a deep breath or a light tap in case of no response [12]. The anaesthesia provider recorded the triggered alarms from the monitor hourly. Foetal heart rate was continuously monitored with CTG (Hewlett Packard

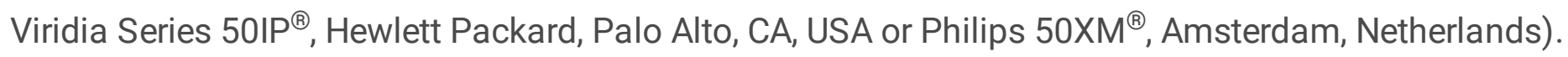
Remifentanil was stopped if pathological CTG changes occurred including decreased variability, bradycardia, tachycardia, or late decelerations.

In the CSEA group, all blocks were performed in the sitting position. The epidural space was located with an 18-gauge Touhy needle (PORTEX ${ }^{\circledR}$ CSE cure ${ }^{\circledR}$ Combined Spinal Epidural System, Smiths Medical, Minnesota, USA) inserted in the midline using loss of resistance to air or saline at the L3-L4 or L4-L5 interspace, followed by needle-through-needle insertion of 27-gauge spinal needle. After obtaining a cerebrospinal fluid, $2.5 \mathrm{mg}$ bupivacaine hydrochloride with $25 \mu \mathrm{g}$ of fentanyl (total volume of $1 \mathrm{ml}$ ) was injected, followed by a 20-gauge multi-hole catheter insertion into the epidural space. Epidural anaesthesia was managed using patient-controlled boluses of $6 \mathrm{ml}^{\circ} 0.1 \%$ bupivacaine with $2 \mu \mathrm{g} \mathrm{ml}^{-1}$ fentanyl every 15 minutes with no background infusion via the PCA pump (Rhythmic ${ }^{\text {TM }}$ Evolution, Micrel Medical Devices, Athens, Greece). In accordance with local protocol, $5 \mathrm{mg}$ of ephedrine was injected into all women immediately after the intrathecal analgesic injection to prevent hypotension.

Demographic and medical data were obtained by means of personal interviews before initiation of analgesia and during the labour. Pain intensity was assessed using an 11-point NRS, where 0 is no pain and 10 the worst imaginable pain. NRS scores, sedation level, automatic readings of non-invasive systolic and diastolic blood pressure, heart rate and $\mathrm{SpO}_{2}$ were recorded immediately before starting the PCA (baseline), every 15 minutes during the first hour and every 30 minutes thereafter. Immediately after delivery, satisfaction with pain relief was evaluated using a five-point categorical scale $(5=$ very good, $4=$ good, 3 = moderate, 2 = poor, 1 = very poor). At the same time, each parturient was also asked if she would choose the same analgesic technique for her next delivery or recommend it to others.

Data on labour progress (first and second stage labour duration, mean cervical dilation rate) and labour outcome were recorded for each patient, including the use of oxytocin, cumulative dose of oxytocin administered, and mode of delivery (spontaneous vaginal, instrumental vaginal, caesarean section). Cervical dilation was assessed by the midwife, and all changes were recorded until the delivery was completed. The mean cervical dilation rate was defined as 10 minus the cervical dilatation observed at the last examination before the start of analgesia divided by the duration of labour.

The number of epidural boluses as well as the total dose of remifentanil were registered automatically in the PCA pump and recorded for each patient. Data concerning nausea, vomiting and itching were also collected. Oral temperature was measured both at the onset of analgesia and within 1 hour of delivery. After delivery, Apgar scores at 1 and 5 minutes were recorded, and umbilical blood gas analysis 
performed according to the standard procedures. Neonatal need for naloxone and resuscitation were also noted.

\section{Statistical analysis}

The Shapiro-Wilk test was used to evaluate the data for normality. If the normality and equal variance assumptions were met, it was followed by Student's t test; otherwise the Mann Whitney U test was used. Proportions were compared using Fisher's exact test. NRS scores at different time points during labour were compared using mixed-effect analysis followed by Sidak post-hoc tests that corrected the $p$ values for the subgroup analyses. Statistical analysis was performed with the GraphPad Prism 8 (GraphPad Software, San Diego CA, USA). The difference was considered statistically significant at $p<0.05$. The data are presented as frequency (proportion \%), mean (standard deviation (SD)) and median [inter-quartile range (IQR)].

The sample size was calculated based on the primary outcome of pain relief during CSEA in multiparous parturients measured on an 11-point scale ranging from 0 to 10. If the true difference between the two studied groups is 1 (on 11-point scale with estimated standard deviation of 2.2), we needed to study 77 subjects in each group to be able to reject the null hypothesis that the population means of the two groups are not equal with probability (power) of 0.8 . The Type I error probability associated with the test of this null hypothesis is 0.05 [13].

\section{Results}

One hundred and sixty-two multiparous women were enrolled in the study. Eighty received RPCA while eighty-two received CSEA. One hundred and sixty-one women delivered vaginally. A caesarean section was performed on one parturient, who was excluded from the analysis, due to dystocia. (Fig. 1) Demographic data and obstetric end points did not differ between the two groups except for one cumulative dose of oxytocin which was higher in the CSEA group (Table 1).

Pain scores were similar at the baseline. After $15 \mathrm{~min}$, pain scores decreased significantly in both groups. After 45 min, pain scores in the RPCA group increased and were no longer statistically different from baseline. By contrast, CSEA related pain scores remained significantly lower compared to baseline throughout the rest of the labour (Table 2). Intergroup comparison showed that the relative decrease in pain scores in the CSEA group was significantly larger in the first 90 minutes of labour compared to the RPCA group (Fig. 2). Additional epidural bolus was required in 66 (81\%) women, with maximal frequency of 7 in two patients.

Satisfaction with pain relief was significantly greater with CSEA as compared to RPCA [3.9 (0.36) vs. 2.6 (1.06), $P<0.001]$. Accordingly, more patients from the CSEA group indicated that they would choose the same technique for their next labour [CSEA; 77 (95\%) vs. RPCA; 65 (81\%), $P=0.003$ ]. In spite of that, more than $80 \%$ of women in the RPCA group graded their satisfaction with pain relief as good and very good. 
No difference was observed in recommending the respective technique to others [CSEA; 77 (95\%) vs. RPCA; 72 (90\%), $P=0.118$ ]. There was no crossover from either group (Fig. 1).

No serious cardiorespiratory adverse events such as the need for bag/mask ventilation or cardiopulmonary resuscitation were noticed in any group. In the RPCA group, desaturation (saturation < $94 \%)$, bradypnea and apnoea occurred in 27 (34\%), 17 (21\%) and $20(25 \%)$ respective cases. All women recovered spontaneously upon verbal or tactile stimulation. Most women were awake (sedation score 1 or 2). Only 2 (2\%) in the RPCA group exhibited sedation score of 3 between 15 and 60 minutes, but they were easily woken up (Table 3 ). Apgar scores were reassuring in all new-borns (>8), both at 1 and 5 minutes after birth. None of the new-borns exhibited umbilical artery $\mathrm{pH}<7.0$.

\section{Discussion}

This study shows that in multiparas, CSEA provides better pain relief than RPCA, a result which reconfirms the superiority of neuraxial analgesia over remifentanil regardless of parity. Pain scores associated with CSEA were significantly lower at each set time-point after treatment, indicating a more sustained efficacious labour analgesia. By contrast, the administration of remifentanil offered only mild pain reduction, which was temporary and most effective during the early stage of labour, with pain scores returning to pre-treatment levels as the labour progressed. It should be noted that this observation does not in itself imply a lack of efficacy, since pain scores are known to increase during labour [14]. Similar pain relief with remifentanil has been observed by other researchers whose bolus dosages used were similar to ours $[5,8,15]$. In contrast, Tveit et al., whose mean bolus doses ranged from 0.30-1.05 $\mu \mathrm{g} \mathrm{kg}^{-1}$, obtained much lower pain scores $(3.8 \pm 1.7) 60$ min after the onset of analgesia on account of severe sedation and increase rate of desaturation. Together those factors suggest a narrow window of analgesic efficacy for remifentanil [11]. The difference in pain perception as a function of parity may have also contributed to the increase in pain scores observed in both our groups 60 minutes after the onset of analgesia. In contrast to primiparas, whose pain is relatively great early in labour, multiparas' pain reports increase dramatically in active and transitional labour, and peak during the second stage of labour due to rapid and abrupt foetal descent [16]. Thus, to improve the efficacy of remifentanil in multiparas, RPCA should be initiated later in labour with a faster dose increase over time $[14,17]$. Moreover, given that $81 \%$ of women in the CSEA group required top-up doses, we may conclude that a single spinal injection cannot replace the CSEA technique in multiparas unless performed in the later stages of labour when the labour expectancy is short.

Despite lower analgesic efficacy, the acceptance of remifentanil was high as the vast majority of multiparas graded their overall satisfaction with pain relief as very good and good with a high rate of recommendation and no cross-overs to neuraxial analgesia. This suggests that even mild pain relief is clinically relevant in patients with a shorter labour duration while reflecting that longer and more complicated labours may require more efficient analgesia $[5,6,8,18]$. The measurement of pain and satisfaction during labour is complex. Satisfaction with pain relief is influenced by many factors, such as continuity, expectations, feeling of control, and the availability of options for pain relief. The latter gives 
women the option of choosing the technique which will best meet their expectations [19]. Women with a normal labour progress or expectations of a quick labour are more likely to choose RPCA to avoid the side-effects of neuraxial techniques, which may further contribute to their general satisfaction [20]. Moreover, we only studied satisfaction with labour analgesia in a very short time-window after delivery. Previous studies on satisfaction with labour experience have shown that women tend to grade their labour experience better immediately after delivery than after a longer postpartum interval, which may be attributed to the "Halo effect" of giving birth influencing the overall birth satisfaction [21, 22]. Further studies investigating long term satisfaction with pain relief during labour are, therefore, needed.

This study reconfirmed the well-known risk of respiratory adverse events associated with RPCA [12]. A similar desaturation rate was also reported by others using threshold and dosage ranges similar to ours $[5,15]$. Nevertheless, after implementing a lower dose range regimen (10-30 $\mu \mathrm{g})$, a decrease in desaturation rate was observed on account of worse pain scores and a lower satisfaction rate. [15, 23]. Weiniger et al. also noticed episodes of apnoea which could not be reliably predicted [9]. Nevertheless, desaturation and apnoea were also noticed during normal labour, possibly reflecting a natural physiological process exacerbated by the concomitant use of opioids or nitrous oxide [24,25]. Further studies investigating the remifentanil safety profile particularly in combination with nitrous oxide would therefore be warranted. In contrast to previous reports $[10,11]$, most women on remifentanil remained awake, coming from our incremental increase of the dose with a ceiling limit of $40 \mu \mathrm{g}$ and a sedation score of 2 . We particularly avoided exceeding these limits in order to prevent serious respiratory adverse events.

As with previous reports, no adverse neonatal outcom11-1e was observed in either group [3-6, 8, 9, 12, 13, 16, 23-27]. On the contrary, when remifentanil was used for induction of general anaesthesia for caesarean section, thoracic rigidity, negative effect on Apgar scores and neonatal ventilation were encountered pointing to a necessity to provide a sufficient time between infusion stopping and cord clamping (at least 5-10 $\mathrm{min}$ ) to allow the drug clearance from the foetal blood [29]. Furthermore, no differences were observed in the duration of first and second stage labours between the groups. However, a higher cumulative dose of oxytocin in the CSEA group may be attributed to its slowing down of labour [30].

Our study has limitations: First, due to its observational nature it could not exclude potential biases and confounders. For instance, pain scores, satisfaction as well as side effects were recorded by a nonblinded observer, which may favour bias. Second, the satisfaction with labour experience, including the pain-relief satisfaction during labour, depends heavily on the progress of labour and maternal as well as neonatal outcomes. This is why we compared progress of labour, need for labour augmentation and perinatal outcomes in the two study groups. Since no differences were observed, the groups may be considered as comparable. Nevertheless, despite the limitations of the study's design, giving women a choice in selecting their pain relief may have contributed significantly to their overall satisfaction. Third, the respiratory data should be taken with caution since the use of continuous capnography is known to be associated with false positive results [9]. 


\section{Conclusions}

In multiparas, CSEA provided better pain relief than RPCA. Despite that, levels of acceptance and approval of remifentanil were high, since the vast majority of women graded their satisfaction with pain relief as good and very good with no crossovers between the groups observed. That could be attributed to the fact that women with previewed shorter labour were more likely to choose a less invasive opioid analgesic option which provided an element of personal control. Thus, given no difference in labour outcomes, remifentanil could be offered as a routine labour analgesic option provided rigorous adherence to protocols are ensured. The presence of a midwife is essential for timely management of respiratory depression or other severe outcomes.

\section{List Of Abbreviations}

ASA American Society of Anaesthesiologists

BMI body mass index

CSEA combined spinal-epidural analgesia

CTG cardiotocography

IQR inter-quartile range

NRT numerical rate score

PCA patient-controlled analgesia

RPCA remifentanil patient-controlled analgesia

SD standard deviation

\section{Declarations}

\section{Ethics approval and consent to participate}

This prospective observational study was approved by the Republic of Slovenia National Medical Ethics Committee (Permit No. 91/04/16) and registered th the central database of ClinicalTrials.gov under the trial ID NCT02963337, (https://clinicaltrials.gov/ct2/show/NCT02963337?term=02963337\&rank=1) on November 15, 2016.

\section{Consent for publication}

Not applicable 
The datasets used and/or analysed during the current study are available from the corresponding author on reasonable request.

\section{Competing interests}

The authors declare that they have no competing interests.

\section{Funding}

This work was supported by the Slovenian Research Agency (grant number P3-0043) and tertiary funding from Clinical department of Anaesthesiology and Intensive Therapy, University Clinical Centre (Ljubljana, Slovenia) (grant number TP 20180063).

\section{Authors' contributions}

TSP, IB, ML designed the study, conducted the study, collected data, wrote the manuscript, accepted the final version; NS, TZ collected data, wrote the manuscript, accepted the final version; NU analysed data, wrote the manuscript, accepted the final version; MVV designed the study, wrote the manuscript, accepted the final version.

\section{Acknowledgments}

We are tankful to dr. Dragica Becanovic, dr. Dragan Graovac and residents from the Department of Anaesthesiology and Intensive Therapy, University Medical Centre Ljubljana for helping recruiting the patients, and to Milan Stevanec for technical support.

\section{References}

1. Kan RE, Hughes SC, Rosen MA, Kessin C, Preston PG, Lobo EP. Intravenous remifentanil: placental transfer, maternal and neonatal effects. Anesthesiology. 1998;88:1467-74.

2. Kapila A, Glass PSA, Jacobs JR, Muir KT, Hermann DJ, Shiraishi M, et al. Measured context-sensitive half-times of remifentanil and alfentanil. Anesthesiology. 1995;83:968-75.

3. Lin R, Tao Y, Yu Y, Xu Z, Su J, Liu Z. Intravenous remifentanil versus epidural ropivacaine with sufentanil for labour analgesia: A retrospective study. PLoS One. 2014;9:e112283.

4. Ismail MT, Hassanin MZ. Neuraxial analgesia versus intravenous remifentanil for pain relief in early labor in nulliparous women. Arch Gynecol Obstet. 2012;286:1375-81.

5. Freeman LM, Bloemenkamp KW, Franssen MT, Papatsonis DN, Hajenius PJ, Hollmann MW, et al. Patient controlled analgesia with remifentanil versus epidural analgesia in labour: Randomised multicentre equivalence trial. BMJ. 2015;350 feb23 10:h846.

6. Logtenberg SLM, Oude Rengerink K, Verhoeven CJ, Freeman LM, van den Akker ESA, Godfried MB, et al. Labour pain with remifentanil patient-controlled analgesia versus epidural analgesia: a randomised equivalence trial. BJOG An Int J Obstet Gynaecol. 2017;124:652-60. 
7. Goodman SR, Smiley RM, Negron MA, Freedman PA, Landau R. A randomized trial of breakthrough pain during combined spinal-epidural versus epidural labor analgesia in parous women. Anesth Analg. 2009;108:246-51.

8. Blair JM, Hill DA, Fee JPH. Patient-controlled analgesia for labour using remifentanil: A feasibility study. Br J Anaesth. 2001;87:415-20.

9. Weiniger CF, Carvalho B, Stocki D, Einav S. Analysis of physiological respiratory variable alarm alerts among laboring women receiving remifentanil. Anesth Analg. 2017;124:1211-8.

10. Tveit TO, Halvorsen A, Seiler S, Rosland JH. Efficacy and side effects of intravenous remifentanil patient-controlled analgesia used in a stepwise approach for labour: An observational study. Int $J$ Obstet Anesth. 2013;22:19-25.

11. Tveit TO, Seiler S, Halvorsen A, Rosland JH. Labour analgesia: A randomised, controlled trial comparing intravenous remifentanil and epidural analgesia with ropivacaine and fentanyl. Eur $J$ Anaesthesiol. 2012;29:129-36.

12. Stocki D, Matot I, Einav S, Eventov-Friedman S, Ginosar Y, Weiniger CF. A randomized controlled trial of the efficacy and respiratory effects of patient-controlled intravenous remifentanil analgesia and patient-controlled epidural analgesia in laboring women. Anesth Analg. 2014;118:589-97.

13. Todd KH, Funk KG, Funk JP, Bonacci R. Clinical significance of reported changes in pain severity. Ann Emerg Med. 1996;27:485-9.

14. Douma MR, Verwey RA, Kam-Endtz CE, Van Der Linden PD, Stienstra R. Obstetric analgesia: A comparison of patient-controlled meperidine, remifentanil, and fentanyl in labour. $\mathrm{Br} \mathrm{J}$ Anaesth. 2010;104:209-15.

15. Melber AA, Jelting Y, Huber M, Keller D, Dullenkopf A, Girard T, et al. Remifentanil patient-controlled analgesia in labour: six-year audit of outcome data of the RemiPCA SAFE Network (2010-2015). Int J Obstet Anesth. 2019.

16. Lowe NK. Parity and pain during parturition. J Obstet Gynecol neonatal Nurs JOGNN. 1987;16:3406.

17. Douma MR, Middeldorp JM, Verwey RA, Dahan A, Stienstra R. A randomised comparison of intravenous remifentanil patient-controlled analgesia with epidural ropivacaine/sufentanil during labour. Int J Obstet Anesth. 2011;20:118-23.

18. Volikas I, Butwick A, Wilkinson C, Pleming A, Nicholson G. Maternal and neonatal side-effects of remifentanil patient-controlled analgesia in labour. Br J Anaesth. 2005;95:504-9.

19. Hodnett ED. Pain and women's satisfaction with the experience of childbirth: A systematic review. Am J Obstet Gynecol. 2002;186 5 SUPPL.:S160-72.

20. Frauenfelder S, Van Rijn R, Radder CM, De Vries MC, Dijksman LM, Godfried MB. Patient satisfaction between remifentanil patient-controlled analgesia and epidural analgesia for labor pain. Acta Obstet Gynecol Scand. 2015;94:1014-21.

21. Conesa Ferrer MB, Canteras Jordana M, Ballesteros Meseguer C, Carrillo García C, Martínez Roche ME. Comparative study analysing women's childbirth satisfaction and obstetric outcomes across 
two different models of maternity care. BMJ Open. 2016;6:e011362.

22. Jha P, Larsson M, Christensson K, Svanberg AS. Satisfaction with childbirth services provided in public health facilities: Results from a cross-sectional survey among postnatal women in Chhattisgarh, India. Glob Health Action. 2017;10:1386932.

23. Birnbach DJ, Ranasinghe JS. Is remifentanil a safe and effective alternative to neuraxial labor analgesia? It all depends. Anesth Analg. 2014;118:491-3.

24. Reed PN, Colquhoun AD, Hanning CD. Maternal oxygenation during normal labour. $\mathrm{Br} J$ Anaesth. 1989;62:316-8.

25. Griffin RP, Reynolds F. Maternal hypoxaemia during labour and delivery: the influence of analgesia and effect on neonatal outcome. Anaesthesia. 1995;50:151-6.

26. Volmanen P, Sarvela J, Akural El, Raudaskoski T, Korttila K, Alahuhta S. Intravenous remifentanil vs. epidural levobupivacaine with fentanyl for pain relief in early labour: A randomised, controlled, double-blinded study. Acta Anaesthesiol Scand. 2008;52:249-55.

27. Douma MR, Stienstra R, Middeldorp JM, Arbous MS, Dahan A. Differences in maternal temperature during labour with remifentanil patient-controlled analgesia or epidural analgesia: a randomised controlled trial. Int J Obstet Anesth. 2015;24:313-22.

28. Messmer AA, Potts JM, Orlikowski CE. A prospective observational study of maternal oxygenation during remifentanil patient-controlled analgesia use in labour. Anaesthesia. 2016;71:171-6.

29. Van de Velde M, Teunkens A, Kuypers M, Dewinter T, Vandermeersch E. General anaesthesia with target controlled infusion of propofol for planned caesarean section: maternal and neonatal effects of a remifentanil-based technique. Int J Obstet Anesth. 2004;13:153-8.

30. Anim-Somuah M, Smyth RM, Cyna AM, Cuthbert A. Epidural versus non-epidural or no analgesia for pain management in labour. Cochrane Database Syst Rev. 2018;2018:CD000331.

\section{Tables}

Table 1 Patient characteristics and obstetric outcomes. 


\begin{tabular}{|c|c|c|c|}
\hline & $\begin{array}{l}\text { CSEA } \\
n=81\end{array}$ & $\begin{array}{l}\text { RPCA } \\
n=80\end{array}$ & $P$-value \\
\hline ASA physical status $(2 / 3)$ & $76 / 5$ & $74 / 6$ & 0.766 \\
\hline Maternal age $(y)$ & $33(5)$ & $32(5)$ & 0.760 \\
\hline Weight (kg) & $81(13)$ & $84(14)$ & 0.147 \\
\hline BMI $\left(\mathrm{kg} \mathrm{m}^{-2}\right)$ & $29.1(4.7)$ & $29.8(5.4)$ & 0.519 \\
\hline Gestational age (weeks) & $39(1)$ & $39(2)$ & 0.364 \\
\hline Parity & $2[2-2]$ & $2[2-2]$ & 0.913 \\
\hline Cervix dilatation at initiation of analgesia $(\mathrm{cm})$ & $4.0(0.8)$ & $4.4(0.6)$ & 0.004 \\
\hline Duration of $1^{\text {st }}$ stage of labour (min) & $90(59)$ & $78(47)$ & 0.156 \\
\hline Duration of $2^{\text {nd }}$ stage of labour (min) & $22(16)$ & 19 (15) & 0.222 \\
\hline Speed of cervical dilation $>1.5 \mathrm{~cm} \mathrm{~h}^{-1}$ & $63(78 \%)$ & $69(86 \%)$ & 0.218 \\
\hline Labour induction & $34(42 \%)$ & $28(35 \%)$ & 0.419 \\
\hline Augmentation with oxytocin & $74(91 \%)$ & $71(89 \%)$ & 0.609 \\
\hline Cumulative oxytocin dose (IU) & $14(8)$ & $11(6)$ & 0.017 \\
\hline Instrumental delivery & $0(0 \%)$ & $0(0 \%)$ & 0.999 \\
\hline Episiotomy & $74(91 \%)$ & $71(89 \%)$ & 0.713 \\
\hline Postpartum haemorrhage $\geq 500 \mathrm{ml}$ & $4(5 \%)$ & $3(4 \%)$ & 0.999 \\
\hline Perineal tear $\geq 3^{\text {rd }}$ degree & $1(1 \%)$ & $0(0 \%)$ & 0.999 \\
\hline Vertex OA position & $77(94 \%)$ & $75(94 \%)$ & 0.720 \\
\hline Vertex OP position & $3(4 \%)$ & $5(6 \%)$ & 0.720 \\
\hline Birth weight (g) & $3509(471)$ & $3469(430)$ & 0.581 \\
\hline Head circumference $(\mathrm{cm})$ & $35.2(1.3)$ & $34.9(1.2)$ & 0.130 \\
\hline Apgar $1 \mathrm{~min}$ & 9 [9-9] & $9[9-9]$ & 0.999 \\
\hline Apgar 5 min & 9 [9-9] & $9[9-9]$ & 0.999 \\
\hline Umbilical artery pH & $7.25(0.09)$ & $7.28(0.08)$ & 0.058 \\
\hline Umbilical vein $\mathrm{pH}$ & $7.32(0.07)$ & $7.34(0.07)$ & 0.233 \\
\hline Foetal bradycardia & $2(2 \%)$ & $4(5 \%)$ & 0.277 \\
\hline
\end{tabular}

Values are number (proportion \%), mean (SD) or median [IQR]. ASA, American Society of Anaesthesiologist's physical status; CSEA, combined spinal and epidural analgesia; RPCA, 
remifentanil patient-controlled analgesia.

Table 2 Numerical rate scale (NRS) scores in multiparous women.

\begin{tabular}{llll}
\hline & CSEA & RPCA & \# P-value \\
\hline NRS at inclusion & $8.0(1.7), \mathrm{n}=81$ & $7.9(1.4), \mathrm{n}=80$ & 0.999 \\
NRS at $15 \mathrm{~min}$ & $1.7(2.5), \mathrm{n}=80^{* *}$ & $6.1(1.7), \mathrm{n}=78^{* *}$ & $<0.0001$ \\
\hline NRS at $30 \mathrm{~min}$ & $1.3(2.2), \mathrm{n}=77^{* *}$ & $6.3(1.9), \mathrm{n}=71^{* *}$ & $<0.0001$ \\
\hline NRS at $45 \mathrm{~min}$ & $1.8(2.3), \mathrm{n}=70^{* *}$ & $6.4(2.0), \mathrm{n}=65^{* *}$ & $<0.0001$ \\
\hline NRS at $60 \mathrm{~min}$ & $2.0(2.1), \mathrm{n}=58^{* *}$ & $7.0(1.9), \mathrm{n}=57^{*}$ & $<0.0001$ \\
\hline NRS at $90 \mathrm{~min}$ & $3.2(2.4), \mathrm{n}=47^{* *}$ & $7.6(1.9), \mathrm{n}=40$ & $<0.0001$ \\
\hline NRS at $120 \mathrm{~min}$ & $5.0(2.2), \mathrm{n}=32^{* *}$ & $8.0(1.9), \mathrm{n}=22$ & $<0.0001$ \\
\hline NRS at $150 \mathrm{~min}$ & $5.8(2.7), \mathrm{n}=19^{*}$ & $8.7(1.3), \mathrm{n}=9$ & 0.008 \\
\hline NRS at $180 \mathrm{~min}$ & $5.4(2.5), \mathrm{n}=10^{*}$ & $7.5(1.0), \mathrm{n}=4$ & 0.370 \\
\hline
\end{tabular}

Values are expressed as means (SD). RPCA, remifentanil patient-controlled analgesia; CSEA, combined spinal-epidural analgesia. \# $p$ values for RPCA vs. CSEA at the same time point; ${ }^{*} P=0.01-0.05,{ }^{* *} P<0.0001 \mathrm{vs}$ NRS score at inclusion of the same study group (mixedeffects analysis with Sidak correction).

Table 3 Drug consumption and adverse effects in multiparous women. 


\begin{tabular}{llll}
\hline & CSEA & RPCA & $P$-value \\
& $\mathrm{n}=81$ & $\mathrm{n}=80$ & \\
\hline Remifentanil cumulative dose $(\mathrm{mg})$ & $\mathrm{NA}$ & $0.600[0.343-1.018]$ & $\mathrm{NA}$ \\
Number of boluses needed & $2[1-3]$ & $22[14-34]$ & $<0.0001$ \\
\hline Pruritus & $18(22 \%)$ & $1(1 \%)$ & 0.999 \\
\hline Nausea or vomiting & $4(5 \%)$ & $4(5 \%)$ & 0.292 \\
\hline Temperature at initiating analgesia $\left({ }^{\circ} \mathrm{C}\right)$ & $36.7(0.3)$ & $36.6(0.3)$ & 0.297 \\
\hline Temperature within 1 hour after delivery $\left({ }^{\circ} \mathrm{C}\right)$ & $37.0(0.4)$ & $36.9(0.5)$ & 0.746 \\
\hline Systolic blood pressure drop $>20 \mathrm{mmHg}^{-1}$ & $6(8 \%)$ & $4(5 \%)$ & 0.999 \\
\hline Heart rate drop below 60 min ${ }^{-1}$ & $1(1 \%)$ & $0(0 \%)$ & \\
\hline Desaturation $($ SpO \\
\hline
\end{tabular}

Values are expressed as an absolute number (proportion \%), mean (SD) or median [IQR]. RPCA, remifentanil patient-controlled analgesia; CSEA, combined spinal-epidural analgesia; NA, not applicable.

\section{Figures}




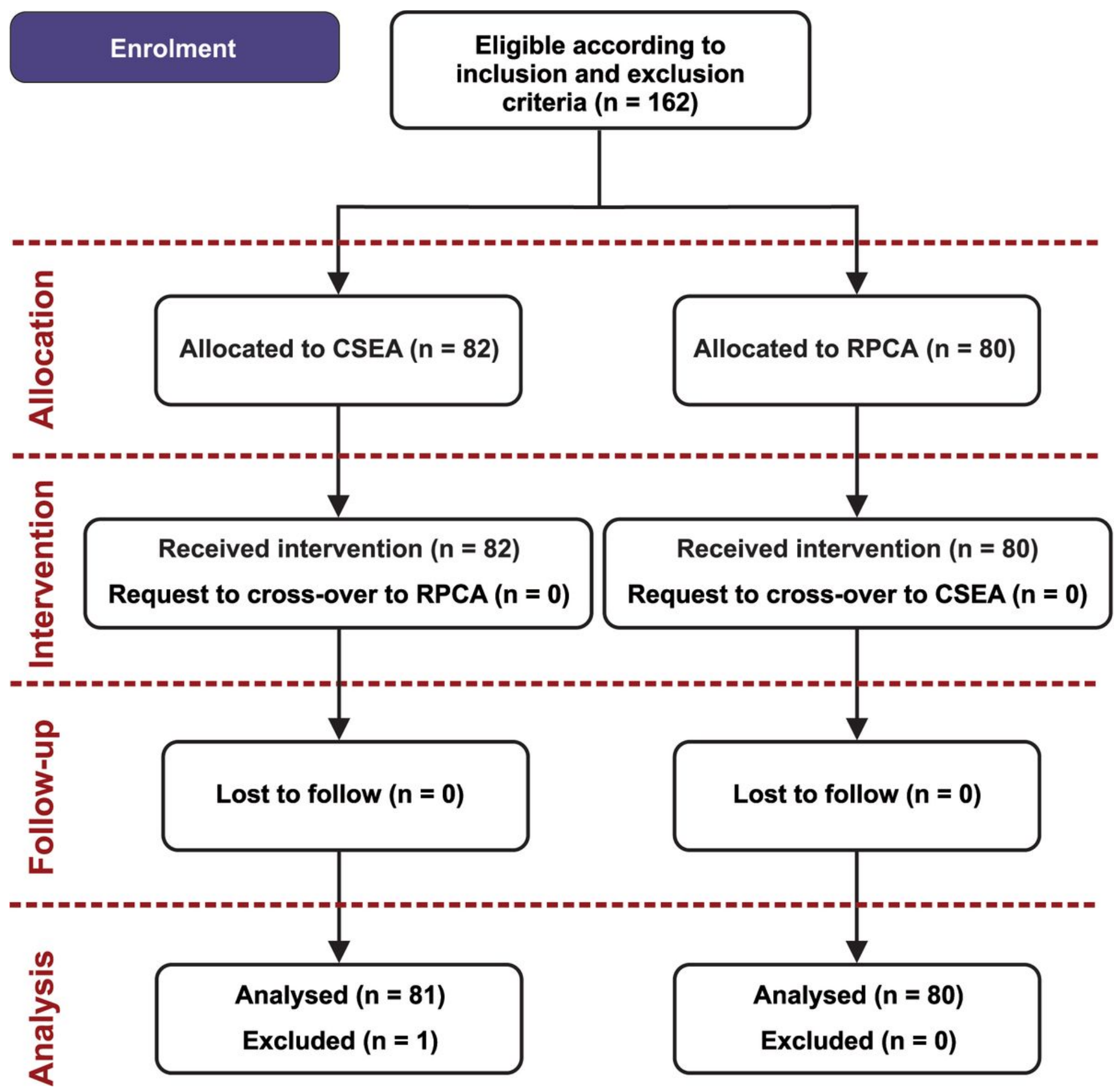

Figure 1

Study design 


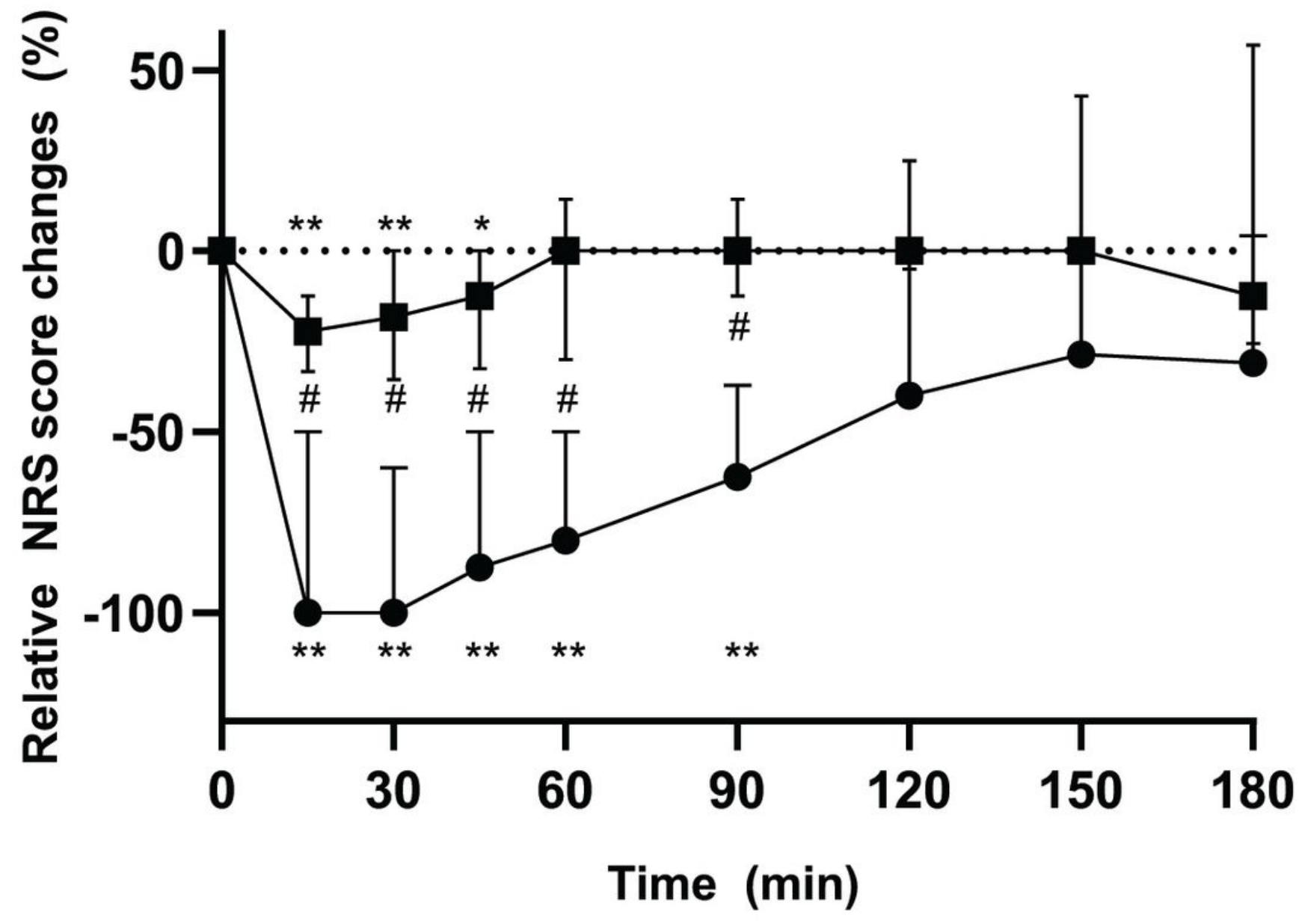

Figure 2

Relative numerical rating scale (NRS) score changes from the baseline value in multiparous women. Remifentanil patient-controlled analgesia (RPCA) ( $\mathbf{\square})$ or combined spinal-epidural analgesia (CSEA) ( $)$ during labour. Data are medians and inter-quartile ranges. ${ }^{*} P<0.01, * * P<0.0001$ vs baseline NRS score of the same study group; $\# \mathrm{P}<0.0001$ relative NRS score changes of the RPCA vs. CSEA study group at the same time point (mixed-effects analysis with Sidak correction) 